\title{
Multi-channel miniaturized electrochemical platform based on microfluidics with serial and quantitative dilution
}

\author{
Hao Wan ${ }^{12^{\star}}$, Jiawei Tu${ }^{1}$, Ping Wang ${ }^{1}$ \\ 1 Key Laboratory for Biomedical Engineering of Ministry of Education, Biosensor National Special \\ Laboratory, Department of Biomedical Engineering, Zhejiang University, Hangzhou, 310027, China, \\ 2 Department of Electrical and Computer Engineering, Michigan State University, East Lansing, MI, \\ 48823, USA. \\ Corresponding author: wh1816@zju.edu.cn
}

\begin{abstract}
:
Electrochemical technique has been widely applied in trace analysis on site due to high sensitivity, low detection limit, low time cost and simple instrumentation. Traditional electrochemical platform generally features drawbacks in limited channels, large size and complicated sensor calibration. This paper presents a multi-channel miniaturized electrochemical platform integrated with microfabricated electrodes and a microfluidic chip with serial and quantitative dilution. To implement serial and quantitative dilution on the microfluidic chip, the microfluidic structure was analogous to an electrical circuit and designed accordingly. The function of the designed structure was validated with COMSOL simulation. Microfabrication technique was used for fabrication of the microfluidic chip and electrode arrays. Four electrode arrays were patterned on a glass substrate to construct four independent electrochemical channels. This miniaturized electrochemical platform was implemented by bonding the microfluidic chip with the electrode arrays, and each channel can be independently used for different diluted samples measurement. This platform would provide the feasibility of high throughput electrochemical analysis and rapid sensor calibration with different diluted samples. The platform was tested in potassium ferricynide to validate the serial dilution functionality for electrochemical sensing.
\end{abstract}

Key words: Lab on chip, electrochemical device, electrode array, serial dilution, multi-channel

\section{Introduction}

Lab-on-a-chip platforms has attracted great interests in many applications for biosensing and chemical sensing due to their outstanding performance in measurement time, portability, flexibility and cost [1]. Microfluidic integration leads to well-defined laminar flow, controllable diffusion and easy implementation for massively parallel analysis [2]. Electrochemical method offers superior capability in high sensitivity, good selectivity, low power consumption and easy miniaturization. A variety of electrochemical sensors has been integrated with microfluidic chips to construct platforms for electrochemical sensing. However, most platforms only provide one channel or several identical channels for sensing. This work presents a multi-channel miniaturized electrochemical platform integrated with four microfabricated electrode arrays for sensing, and an elaborate designed microfluidic chip was integrated for serial dilution, thus enabling measurements of samples with different concentrations for fast calibration and crossvalidation.

\section{Simulation of microfluidic device}

To achieve serial dilution in a four-channel device, inlets for samples and buffer solutions are required, and the buffer solution needs to be split into three branches to mix with samples, thus diluting samples into three different concentrations. A schematic of the multichannel microfluidic for serial dilution is shown in Fig. 1. Branches should be well designed to achieve the desired serial dilution in different channels.

To achieve specific dilution ratios in different working channels, the volumetric flow rate in different buffer branches must be adjusted for mixing and dilution. The hydrodynamic resistance can be analogous to the resistance in an electric circuit. The volumetric flow rate acts as the current, and the pressure difference acts as the voltage. Fig. 2 shows the analogous circuit for the overall microfluidic system presented in Fig. 1. 


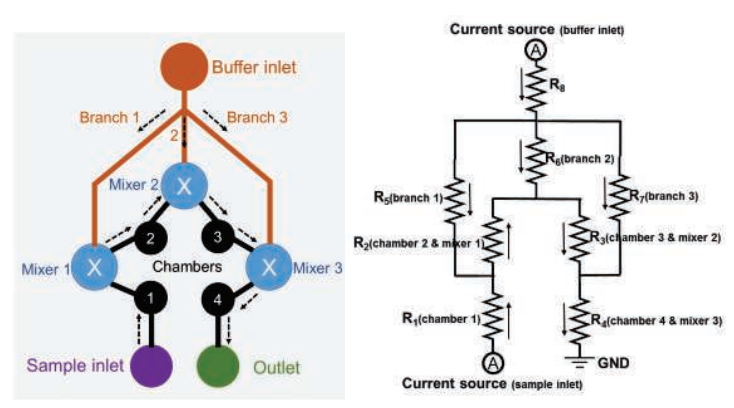

Fig. 1. A schematic of the multi-channel microfluidic for serial dilution (left) and the analogous circuit for the multi-channel microfluidic system(right).

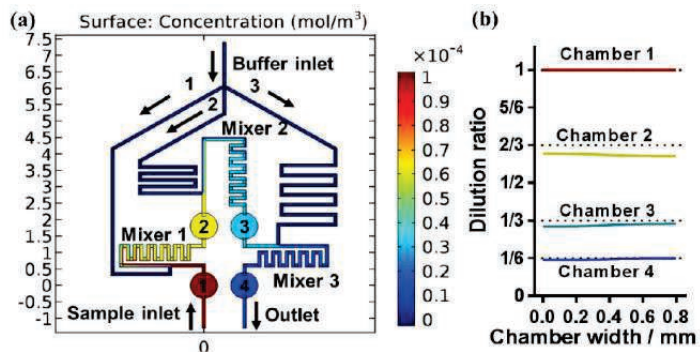

Fig. 2 Concentration profile of the microfluidic device Applying the design principles above to the desired multi-channel microfluidic system in Fig. 1 yields the design shown in Fig. 2(a). The length of the three branches were set to $8.5 \mathrm{~mm}$, $12.95 \mathrm{~mm}$ and $17.38 \mathrm{~mm}$ to achieve desired dilution ratios of $2 / 3,1 / 3$ and $1 / 6$. The concentration distributions in the diameter of four chambers perpendicular to flowing direction were analyzed and shown in Fig. 2(b). Compared to the desired dilution ratios, the relative error is $6.45 \%, 6.15 \%$ and $2.57 \%$. This simulation result indicates that the microfluidic system can achieve desired dilution ratios in different chambers.

\section{Device fabrication and test}

SU-8 photoresist and PDMS were used as the mold material and microfluidic material for device fabrication, respectively. Four electrode arrays comprising three electrodes, were fabricated on a glass substrate using photolithography. The PCB was designed for convenient electrical connection, and the assembled platform is shown in Fig. 3 .

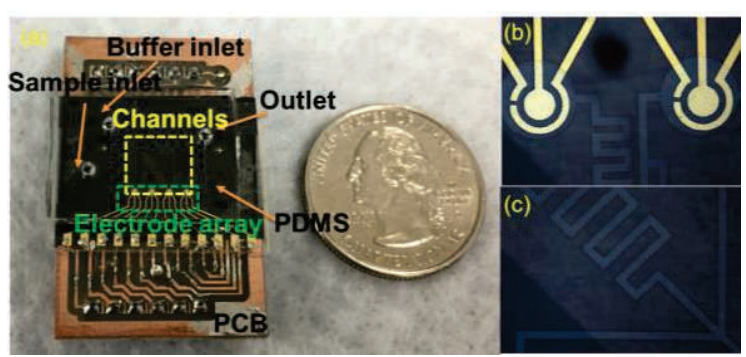

Fig. 3 (a) The assembled electrochemical platform;

(b) zoomed electrode arrays in microfluidic chambers;

(c) zoomed microfluidic channel
Four channels were respectively tested in potassium ferricynide with different scan rates (SR) and concentration. Fig. 4 shows the cyclic voltammograms of one channel under different SR and concentration. The peak current increases with the increasing of SR and concentration, indicating the good functionality of this microfluidic platform. Fig. 4(c) shows the electrochemical responses in four channels, indicating slight different electrochemical characteristics in different channels that may be caused by the fabrication process. Thus, different channels were calibrated individually. The dilution ratio in different channels was calculated as shown in Fig. 4(c), which well matched the simulation results and ideal results. These results well validate the serial dilution and multichannel electrochemical sensing functionality of this platform.
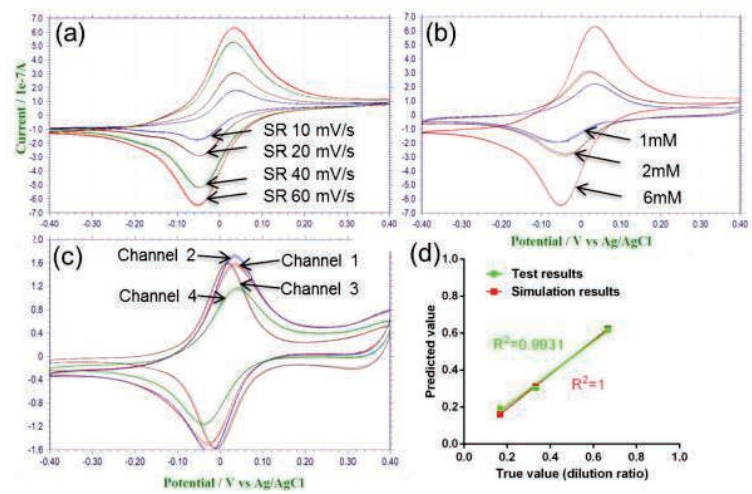

Fig. 4 Electrochemical response of one channel for potassium ferricynide with different $S R$ (a) and concentration (b); (c) comparison of different channels; (d) the calculated dilution ratios compared with simulation results and ideal results.

\section{Conclusion}

This paper presents a multi-channel miniaturized electrochemical platform with serial and quantitative dilution for electrochemical sensing. The structure of the microfluidic channels was well designed and validated using simulation results. This accurate serial dilution of the platform was also validated using electrochemical measurements in potassium ferricynide, indicating a promising platform for fast calibration and cross-validation in electrochemical sensing.

\section{Reference}

[1] G. M. Whitesides, The Origins and the Future of Microfluidics, Nature, 442, 368-373 (2006); doi: 10.1038/nature05058.

[2] M. Pumera, A. Merkoçi, and S. Alegret, New materials for electrochemical sensing VII. Microfluidic chip platforms, TrAC Trends in Analytical Chemistry, 25, 219-235 (2006); doi: 10.1016/j.trac.2005.08.005 